DOI: $10.15393 /$ j3.art.2018.4590

UDC 517.16

J. SÁNdor AND B. A. Bhayo

\title{
ON TWO NEW MEANS OF TWO ARGUMENTS III
}

Abstract. In this paper we establish two sided inequalities for the following two new means

$$
X=X(a, b)=A e^{G / P-1}, \quad Y=Y(a, b)=G e^{L / A-1},
$$

where $A, G, L$ and $P$ are the arithmetic, geometric, logarithmic, and Seiffert means, respectively. As an application, we refine many other well known inequalities involving the identric mean $I$ and the logarithmic mean $L$.

Key words: inequalities, means of two arguments, identric mean, logarithmic mean

2010 Mathematical Subject Classification: 26D05, 26D15, 26D99

1. Introduction. The study of the inequalities involving the classical means such as arithmetic mean $A$, geometric mean $G$, identric mean $I$ and logarithmic mean $L$ have been of the extensive interest for several authors, e.g., see [2, 3, 9, 11, 21, 22, 30, 31, 32, 40.

In 2011, Sándor [27] introduced a new mean $X(a, b)$ for two positive real numbers $a$ and $b$, defined by

$$
X=X(a, b)=A e^{G / P-1},
$$

where $A=A(a, b)=(a+b) / 2, G=G(a, b)=\sqrt{a b}$, and

$$
P=P(a, b)=\frac{a-b}{2 \arcsin \left(\frac{a-b}{a+b}\right)}, \quad a \neq b, \quad P(a, a)=a,
$$

are the arithmetic mean, geometric mean, and Seiffert mean [38, respectively. 
For $p \in \mathbb{R}$ and $a, b>0$ with $a \neq b$, we define the $p$ th power mean $M_{p}(a, b)$ and the $p$ th power-type Heronian mean $H_{p}(a, b)$ by

$$
M_{p}=M_{p}(a, b)= \begin{cases}\left(\frac{a^{p}+b^{p}}{2}\right)^{1 / p}, & p \neq 0, \\ \sqrt{a b}, & p=0\end{cases}
$$

and

$$
H_{p}=H_{p}(a, b)= \begin{cases}\left(\frac{a^{p}+(a b)^{p / 2}+b^{p}}{3}\right)^{1 / p}, & p \neq 0, \\ \sqrt{a b}, & p=0\end{cases}
$$

respectively.

The present paper contains essentially results on the $X$ mean, in particular, several inequalities involving the $X$ mean and the refinements of the following double inequalities are established.

For all $a, b>0$ with $a \neq b$

$$
M_{p}<X<M_{q}
$$

holds if and only if $p \leq 1 / 3$ and $q \geq \log (2) /(1+\log (2)) \approx 0.4094$, and

$$
H_{\alpha}<X<H_{\beta}
$$

holds if and only if $\alpha \leq 1 / 2$ and $\beta \geq \log (3) /(1+\log (2)) \approx 0.6488$.

In the same paper, Sándor [27] introduced another mean $Y(a, b)$ for two positive real $a$ and $b$ by

$$
Y=Y(a, b)=G e^{L / A-1},
$$

where

$$
L=L(a, b)=\frac{a-b}{\log (a)-\log (y)}, \quad a \neq b, \quad L(a, a)=a,
$$

is a logarithmic mean. For two positive real numbers $a$ and $b$, the identric mean and harmonic mean are defined by

$$
I=I(a, b)=\frac{1}{e}\left(\frac{a^{a}}{b^{b}}\right)^{1 /(a-b)}, \quad a \neq b, \quad I(a, a)=a,
$$

and

$$
H=H(a, b)=2 a b /(a+b)
$$


respectively. For the sharp inequalities of logarithmic and identric means, see $([25,[18])$. See also [23], [24], [36]. In 2012, the $X$ mean appeared in [27]. In 2014, $X$ and $Y$ means were published in the journal of Notes on Number Theory and Discrete Mathematics [29. For the historical background and the generalization of these means we refer the reader to, e.g. [3, 9, 17, 21, 22, 28, 30, 31, 32, 33, 34, 40. Connections of these means and the trigonometric or hyperbolic inequalities are given in [5, 27, 29, 32].

In [29], Sándor proved inequalities for $X$ and $Y$ means in terms of other classical means as well as their relationship. Let us recall some of the results for easy reference.

Theorem 1. 29] For $a, b>0$ with $a \neq b$, the following inequalities
1) $G<\frac{A G}{P}<X<\frac{A P}{2 P-G}<P$,
2) $H<\frac{L G}{A}<Y<\frac{A G}{2 A-L}<G$,
3) $1<\frac{L^{2}}{I G}<\frac{L \cdot e^{G / L-1}}{G}<\frac{P X}{A G}$,
4) $H<\frac{G^{2}}{I}<\frac{L G}{A}<\frac{G(A+L)}{3 A-L}<Y$

hold.

In [5] a series expansion of $X$ and $Y$ was presented.

Theorem 2. 5] For $a, b>0$ with $a \neq b$, the following inequalities
1) $\frac{1}{e}(G+H)<Y<\frac{1}{2}(G+H)$,
2) $G^{2} I<I Y<I G<L^{2}$,
3) $\frac{G-Y}{A-L}<\frac{Y+G}{2 A}<\frac{3 G+H}{4 A}<1$,
4) $L<\frac{2 G+A}{3}<X<L(X, A)<P<\frac{2 A+G}{3}<I$,
5) $2\left(1-\frac{A}{P}\right)<\log \left(\frac{X}{A}\right)<\left(\frac{P}{A}\right)^{2}$ 
are true.

Chu et al. 10, and Zhou et al. 41] proved the double inequalities (1) and (2), respectively.

This paper is organized as follows: in Section 1, we give the introduction. Section 2 consists of main results and remarks. In Section 3, some connections of $X, Y$ and other means are given with trigonometric and hyperbolic functions. Some lemmas are also proved in this section which will be used in the proof of the main result. Section 4 deals with the proof of the main result and corollaries. In the computations we have used also the Mathematica software (see e.g.[26]).

2. Main result and motivation. Making contribution to the topic, we refine some previous results appeared in the literature [1, 2, 5, $10,41,29$. as well as establish new results involving the $X$ mean.

Theorem 3. For $a, b>0$

$$
\alpha G+(1-\alpha) A<X<\beta G+(1-\beta) A,
$$

with the best possible constants $\alpha=2 / 3 \approx 0.6667$ and $\beta=(e-1) / e \approx$ $\approx 0.6321$, and

$$
A+G-\alpha_{1} P<X<A+G-\beta_{1} P
$$

with the best possible constants $\alpha_{1}=1$ and $\beta_{1}=\pi(e-1) /(2 e) \approx 0.9929$. Remark. In [29, Theorem 2.7], Sándor proved that for $a \neq b$,

$$
X<A\left[\frac{1}{e}+\left(1-\frac{1}{e}\right) \frac{G}{P}\right]
$$

and

$$
Y<G\left[\frac{1}{e}+\left(1-\frac{1}{e}\right) \frac{L}{A}\right] .
$$

As $A / P>1$, the right side of (3) gives a slight improvement to (5). From (6), as clearly $G \cdot L / A<A$, we get a similar inequality. The second inequality in (4) could be a counterpart of the inequality $L+G-A<Y$ studied in [5, Theorem 20].

H. Alzer [1] proved the following inequalities:

$$
1<(A+G) /(L+I)<e / 2,
$$


where the constants 1 and $2 / e$ are the best possible ones. The following result improves among others the right side of (7).

Theorem 4. For $a \neq b$

$$
(A+G) / e<X<M_{q}<(L+I) / 2<(A+G) / 2,
$$

where $q=\log (2) /(1+\log (2)) \approx 0.4094$ is the best possible constant.

Remark. Particularly, (8) implies that

$$
X<(L+I) / 2
$$

which is new. Since $L<X<I$ (see Theorems 1 and 2), $X$ is less than the arithmetic mean of $L$ and $I$. In fact, by left side of (1), and by $L<M_{1 / 3}$ (see [25], [16]), and $L<I<M_{2 / 3}$ (see [25]; see also [30], for other references), we get also

$$
L<M_{1 / 3}<X<M_{q}<(L+I) / 2<I<M_{2 / 3}
$$

Theorem 5. For $a \neq b$

$$
A+G-P<X<P^{2} / A<(A+G) / 2 .
$$

Remark. The right hand side of (11) offers another refinement to $X<$ $<(A+G) / 2$. An improvement of $P^{2}>X A$ appears in [29, Theorem 2.9]:

$$
P^{2}>\left(A^{2}((A+G) / 2)^{4}\right)^{1 / 3}>A X,
$$

so (11) could be further refined. For the following inequalities

$$
\begin{aligned}
L<\frac{2 G+A}{3} & <A+G-P<X<\sqrt{P X}<\frac{A+G}{2}< \\
< & \frac{P+X}{2}<P<\frac{2 A+G}{3}<I,
\end{aligned}
$$

one can see that the first inequality is Carlson's inequality, while the second written in the form $P<(2 A+G) / 3$ is due to Sándor [33]. The third inequality is Theorem 2.10 in [29], while the fourth, written as $P X<$ $<((A+G) / 2)^{2}$ is Theorem 2.11 of $[29$. The inequality $(P+X) / 2<P$ follows by $X<P$, while the last two inequalities are due to Sándor ([33, 31]). 
Theorem 6. For $a \neq b$

$$
M_{1 / 2}<(P+X) / 2<M_{k},
$$

where $k=(5 \log 2+2) /(6(\log 2+1)) \approx 0.5380$.

Remark. One has

$$
L<\frac{2 G+A}{3}<X<\frac{L+I}{2}<\frac{A+G}{2}<\frac{P+X}{2}<P<\frac{2 A+G}{3}<I
$$

and

$$
\sqrt{A G}<\sqrt{P X}<\frac{A+G}{2} .
$$

Inequalities (15) show that $\sqrt{P X}$ lies between the geometric and arithmetic means of $A$ and $G$, while $(12)$ shows among others that $(A+G) / 2$ lies between the geometric and arithmetic means of $P$ and $X$.

Theorem 7. The following inequalities

$$
\begin{array}{ll}
M_{p} \leq M_{1 / 3}<(2 G+A) / 3<X, & \text { for } \quad p \leq 1 / 3, \\
H_{\alpha} \leq H_{1 / 2}<(2 G+A) / 3<X, & \text { for } \quad \alpha \leq 1 / 2,
\end{array}
$$

hold.

Theorem 8. For $a \neq b$

$$
(A X)^{1 / \alpha_{2}}<P<\left(A X^{\beta_{2}}\right)^{1 /\left(1+\beta_{2}\right)}
$$

with the best possible constants $\alpha_{2}=2$ and $\beta_{2}=\log (\pi / 2) / \log (2 e / \pi) \approx$ $\approx 0.8234$.

3. Preliminaries and lemmas. We use the following result by Biernacki and Krzyż [8] in studying the monotonicity of certain power series.

Lemma 1. Let $A(x)=\sum_{n=0}^{\infty} a_{n} x^{n}$ and $C(x)=\sum_{n=0}^{\infty} c_{n} x^{n}$ be two real power series converging on the interval $(-R, R), 0<R \leq \infty$. If the sequence $\left\{a_{n} / c_{n}\right\}$ is increasing (decreasing) and $c_{n}>0$ for all $n$, then the function $A(x) / C(x)$ is also increasing (decreasing) on $(0, R)$.

For $|x|<\pi$, the following power series expansions

$$
x \cot x=1-\sum_{n=1}^{\infty} \frac{2^{2 n}}{(2 n) !}\left|B_{2 n}\right| x^{2 n},
$$




$$
\begin{aligned}
\cot x & =\frac{1}{x}-\sum_{n=1}^{\infty} \frac{2^{2 n}}{(2 n) !}\left|B_{2 n}\right| x^{2 n-1}, \\
\operatorname{coth} \mathrm{x} & =\frac{1}{x}+\sum_{n=1}^{\infty} \frac{2^{2 n}}{(2 n) !}\left|B_{2 n}\right| x^{2 n-1},
\end{aligned}
$$

can be found in [13, 1.3.1.4 (2)-(3)]. Here $B_{2 n}$ are the even-indexed Bernoulli numbers (see [12, p. 231]). We get the following expansions directly from 19 and 20 .

$$
\begin{aligned}
& \frac{1}{(\sin x)^{2}}=-(\cot x)^{\prime}=\frac{1}{x^{2}}+\sum_{n=1}^{\infty} \frac{2^{2 n}}{(2 n) !}\left|B_{2 n}\right|(2 n-1) x^{2 n-2}, \\
& \frac{1}{(\sinh x)^{2}}=-(\operatorname{coth} x)^{\prime}=\frac{1}{x^{2}}-\sum_{n=1}^{\infty} \frac{2^{2 n}}{(2 n) !}(2 n-1)\left|B_{2 n}\right| x^{2 n-2} .
\end{aligned}
$$

For the following expansion formula

$$
\frac{x}{\sin x}=1+\sum_{n=1}^{\infty} \frac{2^{2 n}-2}{(2 n) !}\left|B_{2 n}\right| x^{2 n}
$$

see 15 .

For easy reference we recall the following lemma from [5, 6].

Lemma 2. For $x=\arcsin ((a-b) /(a+b))$ and $y=(1 / 2) \log (a / b)$, with $a>b>0$, one has

$$
\begin{gathered}
\frac{P}{A}=\frac{\sin (x)}{x}, \frac{G}{A}=\cos (x), \frac{H}{A}=\cos (x)^{2}, \frac{X}{A}=e^{x \cot (x)-1}, \\
\frac{L}{G}=\frac{\sinh (y)}{y}, \frac{L}{A}=\frac{\tanh (y)}{y}, \frac{H}{G}=\frac{1}{\cosh (y)}, \frac{Y}{G}=e^{\tanh (y) / y-1}, \\
\log \left(\frac{I}{G}\right)=\frac{A}{L}-1, \quad \log \left(\frac{Y}{G}\right)=\frac{L}{A}-1 .
\end{gathered}
$$


Remark. It is well known that many inequalities involving the means can be obtained from the classical inequalities for trigonometric functions. For example, the following inequality

$$
e^{(x / \tanh (x)-1) / 2}<\frac{\sinh (x)}{x}, \quad x>0,
$$

recently appeared in [7, Theorem 1.6], is equivalent to

$$
\frac{\sinh (x)}{x}>e^{x / \tanh (x)-1} \frac{x}{\sinh (x)}
$$

By Lemma 2, this can be written as

$$
\frac{L}{G}>\frac{I}{G} \cdot \frac{G}{L}=\frac{I}{L}
$$

or

$$
L>\sqrt{I G} .
$$

The inequality (25) was proved by Alzer [3].

The following trigonometric inequalities (see [7, Theorem 1.5]) imply an other double inequality for Seiffert mean $P$,

$$
\left\{\begin{array}{l}
\exp \left(\frac{1}{2}\left(\frac{x}{\tan x}-1\right)\right)<\frac{\sin x}{x}<\exp \left(\left(\log \frac{\pi}{2}\right)\left(\frac{x}{\tan x}-1\right)\right) \quad x \in(0, \pi / 2), \\
\sqrt{A X}<P<A\left(\frac{X}{A}\right)^{\log (\pi / 2)} .
\end{array}\right.
$$

The second mean inequality in (26) was also pointed out by Sándor (see [29, Theorem 2.12]). For various related trigonometric and hyperbolic inequalities, see also [14], [19].

Lemma 3. 4, Theorem 2] For $-\infty<a<b<\infty$, let $f, g:[a, b] \rightarrow \mathbb{R}$ be continuous on $[a, b]$, and differentiable on $(a, b)$. Let $g^{\prime}(x) \neq 0$ on $(a, b)$. If $f^{\prime}(x) / g^{\prime}(x)$ is increasing (decreasing) on $(a, b)$, then so are

$$
\frac{f(x)-f(a)}{g(x)-g(a)} \quad \text { and } \quad \frac{f(x)-f(b)}{g(x)-g(b)} .
$$

If $f^{\prime}(x) / g^{\prime}(x)$ is strictly monotone, then the monotonicity in the conclusion is also strict.

Lemma 4. The following function

$$
h(x)=\frac{\log (x / \sin (x))}{\log \left(e^{1-x / \tan (x)} \sin (x) / x\right)}
$$


is strictly decreasing from $(0, \pi / 2)$ onto $\left(\beta_{2}, 1\right)$, where

$$
\beta_{2}=\log (\pi / 2) / \log (2 e / \pi) \approx 0.8234 .
$$

In particular, for $x \in(0, \pi / 2)$ we have

$$
\left(\frac{e^{1-x / \tan (x)} \sin (x)}{x}\right)^{\beta_{2}}<\frac{x}{\sin (x)}<\left(\frac{e^{1-x / \tan (x)} \sin (x)}{x}\right) .
$$

Proof. Let

$$
h(x)=\frac{h_{1}(x)}{h_{2}(x)}=\frac{\log (x / \sin (x))}{\log \left(e^{1-x / \tan (x) \sin (x) / x}\right)}
$$

for $x \in(0, \pi / 2)$. Differentiating with respect to $x$ we get

$$
\frac{h_{1}^{\prime}(x)}{h_{2}^{\prime}(x)}=\frac{1-x / \tan (x)}{(x / \sin (x))^{2}-1}=\frac{A_{1}(x)}{B_{1}(x)} .
$$

Using the expansion formula we have

$$
A_{1}(x)=\sum_{n=1}^{\infty} \frac{2^{2 n} 2 n}{(2 n) !}\left|B_{2 n}\right| x^{2 n}=\sum_{n=1}^{\infty} a_{n} x^{2 n}
$$

and

$$
B_{1}(x)=\sum_{n=1}^{\infty} \frac{2^{2 n} 2 n}{(2 n) !}\left|B_{2 n}\right|(2 n-1) x^{2 n}=\sum_{n=1}^{\infty} b_{n} x^{2 n} .
$$

Let $c_{n}=a_{n} / b_{n}=1 /(2 n-1)$, which is the decreasing in $n \in \mathbb{N}$. Thus, by Lemma $11 h_{1}^{\prime}(x) / h_{2}^{\prime}(x)$ is strictly decreasing in $x \in(0, \pi / 2)$. In turn, this implies by Lemma 3 that $h(x)$ is strictly decreasing in $x \in(0, \pi / 2)$. Applying L'Hôpital rule, we get $\lim _{x \rightarrow 0} h(x)=1$ and $\lim _{x \rightarrow \pi / 2} h(x)=\beta_{2}$. This completes the proof.

Remark. It is observed that the inequalities in (27) coincide with the trigonometric inequalities given in $(26)$. Here Lemma 4 gives a new and an optimal proof for these inequalities.

Lemma 5. The following function

$$
f(x)=\frac{1-e^{x / \tan (x)-1}}{1-\cos (x)}
$$


is strictly decreasing from $(0, \pi / 2)$ onto $((e-1) / e, 2 / 3)$ where $(e-1) / e \approx$ $\approx 0.6321$. In particular, for $x \in(0, \pi / 2)$, we have

$$
\frac{1}{\log (1+(e-1) \cos (x))}<\frac{\tan (x)}{x}<\frac{1}{1+\log ((1+2 \cos (x)) / 3)} .
$$

Proof. Write $f(x)=f_{1}(x) / f_{2}(x)$, where $f_{1}(x)=1-e^{x / \tan (x)-1}$ and $f_{2}(x)=1-\cos (x)$ for all $x \in(0 \pi / 2)$. Clearly, $f_{1}(x)=0=f_{2}(x)$. Differentiating with respect to $x$, we get

$$
\frac{f_{1}^{\prime}(x)}{f_{2}^{\prime}(x)}=\frac{e^{x / \tan (x)-1}}{\sin (x)^{3}}\left(\frac{x}{\sin (x)^{2}}-\frac{\cos (x)}{\sin (x)}\right)=f_{3}(x) .
$$

Again

$$
f_{3}^{\prime}(x)=-\frac{e^{x / \tan (x)-1}}{\sin (x)^{3}}(c(x)-2)
$$

where

$$
c(x)=x\left(\frac{\cos (x)}{\sin (x)}+\frac{x}{\sin (x)^{2}}\right) .
$$

In order to show that $f_{3}^{\prime}<0$, it is enough to prove that

$$
c(x)>2,
$$

which is equivalent to

$$
\frac{\sin (x)}{x}<\frac{x+\sin (x) \cos (x)}{2 \sin (x)} .
$$

Applying the Cusa-Huygens 20] inequality

$$
\frac{\sin (x)}{x}<\frac{\cos (x)+2}{3}
$$

we get

$$
\frac{\cos (x)+2}{3}<\frac{x+\sin (x) \cos (x)}{2 \sin (x)}
$$

which is equivalent to $(\cos (x)-1)^{2}>0$. Thus $f_{3}^{\prime}>0$, clearly $f_{1}^{\prime} / f_{2}^{\prime}$ is strictly decreasing in $x \in(0, \pi / 2)$. By Lemma 3, we conclude that the function $f(x)$ is strictly decreasing in $x \in(0, \pi / 2)$. The limiting values follow easily. This completes the proof of the lemma. 
Lemma 6. The following function

$$
f_{4}(x)=\frac{\sin (x)}{x\left(\cos (x)-e^{x \cot (x)-1}+1\right)}
$$

is strictly increasing from $(0, \pi / 2)$ onto $(1, c)$, where $c=2 e /(\pi(e-1)) \approx$ $\approx 1.0071$. In particular, for $x \in(0, \pi / 2)$ we have

$$
1+\cos (x)-e^{x / \tan (x)-1}<\frac{\sin (x)}{x}<c\left(1+\cos (x)-e^{x / \tan (x)-1}\right) .
$$

Proof. Differentiating with respect to $x$ we get

$$
f_{4}^{\prime}(x)=\frac{e(x-\sin (x))\left(e \cos (x)-(x+\sin (x)) e^{x \cot (x)} \csc (x)+e\right)}{x^{2}\left(e \cos (x)-e^{x \cot (x)}+e\right)^{2}} .
$$

Let $f_{5}(x)=\log \left((x+\sin (x)) e^{x \cot (x)} / \sin (x)\right)-\log (e \cos (x)+e)$ for $x \in$ $(0, \pi / 2)$. Differentiation yields

$$
f_{5}^{\prime}(x)=\frac{2-x\left(\cot (x)+x \csc ^{2}(x)\right)}{x+\sin (x)},
$$

which is negative by the proof of Lemma 5, and $\lim _{x \rightarrow 0} f_{5}(x)=0$. This implies that $f_{4}^{\prime}(x)>0$, and $f_{4}(x)$ is strictly increasing. The limiting values follow easily. This implies the proof.

Lemma 7. For $a \neq b$, one has

$$
M_{1 / 3}<(2 G+A) / 3 .
$$

Proof. Let $G=G(a, b)$, etc. Divide both sides with $b$ and put $a / b=x$. Then inequality $(28)$ becomes the following:

$$
\left(\frac{x^{1 / 3}+1}{2}\right)^{3}<4(x+4 \sqrt{x}+1) .
$$

Let $x=t^{6}$, where $t>1$. Then raising both sides of $(29)$ to 3 th power, after elementary transformations we get,

$$
t^{6}-9 t^{4}+16 t^{3}-9 t^{2}+1>0
$$

which can be written as $(t-1)^{4}\left(t^{2}+4 t+1\right)>0$, so it is true. Thus 29 ) and (28) are proved. 
Since $L<M_{1 / 3}$, by $(28)$ we get a new proof , as well as a refinement of Carlson's inequality $L<(2 G+A) / 3$.

Lemma 8. The inequality

$$
H_{1 / 2}<(2 G+A) / 3
$$

holds for $a \neq b$.

Proof. By definition of $H_{\alpha}$ one has

$$
H_{1 / 2}=\left(\left(\sqrt{a}+(a b)^{1 / 4}+\sqrt{b}\right) / 3\right)^{2}=(\sqrt{2(A+G)}+\sqrt{G})^{2} / 9,
$$

by remarking that $\sqrt{a}+\sqrt{b}=\sqrt{2(A+G)}$. Therefore, (2) can be written equivalently as

$$
(2(A+G)+2 \sqrt{2 G(A+G)}+G) / 9<(2 G+A) / 3 .
$$

Now, it is immediate that 31 becomes, after elementary computations

$$
A+3 G>2 \sqrt{2 G(A+G)},
$$

or by raising both sides to the 2 th power:

$$
A^{2}+6 A G+9 G^{2}>8 A G+8 G^{2},
$$

which becomes $(A-G)^{2}>0$, true. Thus $(32)$ and $(31)$ are proved, and (30) follows.

\section{Proof of main result.}

Proof of Theorem 3. By Lemma 5

$$
\frac{e-1}{e}<\frac{1-1 / e^{1-x / \tan (x)}}{\cos (x) / e^{1-x / \tan (x)}-1 / e^{1-x / \tan (x)}}<\frac{2}{3} .
$$

Now we get the proof of (3) by utilizing the Lemma 2. The proof of (4) follows easily from Lemmas 2 and 5 .

Proof of Theorem 4. The second inequality of 8 is the right hand side of (1). In [2], Alzer and Qiu proved the third inequality of (8). The last inequality is the left side of (7). By [10] and [2], $q$ is the best possible constant in both sides. 
Now let us prove the first inequality of (8). By Lemma 2 this becomes equivalent to $1+\cos (x)<e^{x \cot (x)}$, or

$$
\log (1+\cos (x))<x \cot (x), \quad x \in(0, \pi / 2) .
$$

Now, by the classical inequality $\log (1+t)<t \quad(t>0)$, applied to $t=\cos (x)$, we get $\log (1+\cos (x))<\cos (x)$. Now $\cos (x)<x \cot (x)=$ $=x \cos (x) / \sin (x)$ is true by $\sin (x)<x$. The proof of $(33)$ follows.

One has the following relation, in analogy with relation (7) of Theorem 2 for the mean $Y$ :

Corollary. The inequality $(A+G) / e<X<(A+G) / 2$ holds. The constants $e$ and 2 are the best possible ones.

The inequalities $(A+G) / e<X$ and $(2 G+A) / 3<X$ are not comparable.

Proof of Theorem 5. The second inequality of (11) appeared in [27] in the form $P^{2}>A X$. The last inequality follows by $P<(2 A+G) / 3$. Indeed, one has $((2 A+G) / 3)^{2}<A(A+G) / 2$ becomes $2 G^{2}<A^{2}+A G$, and this is true by $G<A$.

Proof of Theorem 6. By [29, Theorem 2.10], one has $P+X>A+G$, and remarking that $(A+G) / 2=M_{1 / 2}$, the left side of 13 follows. For the right hand side of (13), we will use $P<M_{t}$ with $t=2 / 3$ (see [33]), and $X<M_{q}([10])$, where $q=(\log 2) /(\log 2+1)$. On the other hand the function $f(t)=M_{t}$ is known to be strictly log-concave for $t>0$ (see [35]). Particularly, this implies that $f(t)$ is strictly concave. Thus $\left(M_{t}+M_{q}\right) / 2<M_{(t+q) / 2}$. As $(t+q) / 2=k \approx 0.5380$, the result follows.

Corollary. One has the following two sets of inequalities:

1) $P X>P L>A G$,

2) $I L>P L>A G$.

Proof. The first inequality of (1) follows by $X>L$, while the second appears in 33. The first inequality of (2) follows by $I>P$, while the second one is the same as the second one in (1).

Remark. Particularly in Corollary , (2) improves Alzer's inequality $I L>$ $>A G$. Inequality (1) improves $P X>A G$, which appears in [29].

Corollary. One has 
1) $X>A(P+G) /(3 P-G)>(2 G+A) / 3>L$,

2) $P^{2} / A>X>(P+G) / 2$.

Proof. The first two inequalities of (1) appear in [29, Theorem 2.5 and Remark 2.3]. The second inequality of (2) follows by the first inequality of (1) and the remark that $A /(3 P-G)>1 / 2$, since this is $P<(2 A+G) / 3$; while the first one is $P^{2}>A X([27])$.

Remark. Since it is known that $P>(2 / \pi) A$ (due to Seiffert, see [33]). By $X>(P+G) / 2$ we get the inequality $X>[(2 / \pi) A+G] / 2$, which is not comparable with $(A+G) / e<X$.

Proof of Theorem 7. The first inequality of (16) follows, since the function $f(t)=M_{t}$ is known to be strictly increasing. The second inequality follows by (28), while the third one can be found in Theorem 2 .

It is known that $H_{p}$ is an increasing function of $p$. Therefore, the proof of (17) follows by 30 .

Corollary. For $a, b>0$ with $a \neq b$, we have

$$
\frac{I}{L}<\frac{L}{G}<1+\frac{G}{H}-\frac{I}{G} .
$$

Proof. The first inequality is due to Alzer [3], while the second inequality follows from the fact that the function

$$
x \mapsto \frac{1-e^{x / \tanh (x)-1}}{1-\cosh (x)}:(0, \infty) \rightarrow(0,1)
$$

is strictly decreasing. The proof of the monotonicity of the function is the analogue to the proof of Lemma 5 .

The right hand side of (34) may be written as $L+I<G+A$ (by $H=G^{2} / A$ ), and this is due to Alzer (see [2, 30] for history of early results).

Proof of Theorem 8, The proof follows easily from Lemma 4 .

In 37] (see also [39]), Seiffert proved that

$$
\frac{2}{\pi} A<P
$$


for all $a, b>0$ with $a \neq 0$. As a counterpart of the above result we give the following inequalities.

Corollary. The following inequalities

$$
\frac{1}{e} A<\frac{\pi}{2 e} P<X<P
$$

hold true for $a, b>0$ with $a \neq b$.

Proof. The first inequality follows from (35). For the proof of the second inequality we write by Lemma 2

$$
f_{5}^{\prime}(x)=\frac{X}{P}=\frac{x e^{x / \tan (x)-1}}{\sin (x)}=f_{5}(x)
$$

for $x \in(0, \pi / 2)$. Differentiation gives

$$
\frac{e^{x / \tan (x)-1}}{\sin (x)}\left(1-\frac{x^{2}}{\sin (x)^{2}}\right)<0 .
$$

Hence the function $f_{5}$ is strictly decreasing in $x$, with

$$
\lim _{x \rightarrow 0} f_{5}(x)=1 \quad \text { and } \quad \lim _{x \rightarrow \pi / 2} f_{5}(x)=\pi /(2 e) \approx 0.5779 .
$$

This implies the proof.

We finish this paper by giving the following open problem and a conjecture.

Open problem. What are the best positive constants $a$ and $b$, such that

$$
M_{a}<(P+X) / 2<M_{b} .
$$

Conjecture. For $a \neq b$, one has $P X>I L$.

\section{References}

[1] Alzer H. Ungleichungen für Mittelwerte. Arch.Math.(Basel), 1986, 47, pp. 422-426.

[2] Alzer H., Qiu S.-L. Inequalities for means in two variables. Arch. Math., 2003, vol. 80, no. 2, pp. 201-215. 
[3] Alzer H. Two inequalities for means. C. R. Math. Rep. Acad. Sci. Canada, 1987, vol. 9, pp. 11-16.

[4] Anderson G. D., Vamanamurthy M. K., Vuorinen M. Monotonicity Rules in Calculus. Amer. Math. Monthly, 2006, vol. 113, no. 9, pp. 805-816.

[5] Bhayo B. A., Sándor J. On two new means of two variables II., Notes Number Th. Discr. Math., 2014, vol. 20, no. 4, pp. 1-10.

[6] Bhayo B. A., Sándor J. On some inequalities for the identric, logarithmic and related means. J. Math. Ineq., 2015, vol. 9, no. 3, pp. 889-896.

[7] Bhayo B. A., Sándor J. On certain old and new trigonometric and hyperbolic inequalities. Analysis Mathematica, 2015, vol. 41, Issue 1-2, pp. 3-15. DOI: 1007/s10476-015-0102-9.

[8] Biernacki M., Krzyż J. On the monotonicity of certain functionals in the theory of analytic functions. Ann. Univ. Mariae Curie-Skodowska, 1955, vol. 2, pp. 134-145. DOI: 10.4153/CMB-1967-074-9.

[9] Carlson B. C. The logarithmic mean. Amer. Math. Monthly, 1972, vol. 79, pp. 615-618.

[10] Chu Y.-M., Yang Z.-H., Wu L.-M. Sharp power mean bounds for Sándor mean. Abstr. Appl. Anal., 2015, Art. ID 172867, pp. 5.

[11] Hästö P. A. Optimal inequalities between Seiffert's mean and power means. Math. Inequal. Appl., 2004, vol. 1, no. 7, pp. 47-53.

[12] Ireland K., Rosen M. A Classical Introduction to Modern Number Theory. 2nd ed., Springer-Verlag, New York, Berlin, Heidelberg, 1990.

[13] Jeffrey A. Handbook of Mathematical Formulas and Integrals. 3rd ed., Elsevier Academic Press, 2004.

[14] Klén R., Visuri M., Vuorinen M. On Jordan type inequalities for hyperbolic functions. J. Ineq. Appl., 2010, pp. 14. DOI:10.1155/2010/362548.

[15] Li J.-L. An identity related to Jordans inequality. Internat. J. Math. Math. Sci., 2006, vol. 6, Article ID 76782. DOI:10.1155/IJMMS/2006/76782.

[16] Lin T. P. The power mean and the logarithmic mean. Amer. Math. Monthly, 1974, vol. 81, pp. 879-883.

[17] Mitrinović D. S. Analytic Inequalities. Springer-Verlag, Berlin, 1970.

[18] Mitrinović D. S, Pecaric J. E, Fink A. M. Classical and new inequalities in analysis. Kluwer Acad. Publ., 1993, p. 740.

[19] Neuman E., Sándor J. Optimal inequalities for hyperbolic and trigonometric functions. Bull. Math. Anal. Appl., 2011, vol. 3, no. 3, pp. 177-181.

[20] Neuman E., Sándor J. On some inequalities involving trigonometric and hyperbolic functions with emphasis on the Cusa-Huygens, Wilker, and Huygens inequalities. Math. Inequal. Appl., 2010, vol. 13, no. 4, pp. 715-723. 
[21] Neuman E., Sándor J. On the Schwab-Borchardt mean. Math. Pannonica, 2003, vol. 14, no. 2, pp. 253-266.

[22] Neuman E., Sándor J. On the Schwab-Borchardt mean II. Math. Pannonica, 2006, vol. 17, no. 1, pp. 49-59.

[23] Neuman E., Sándor J. Companion inequalities for certain bivariate means. Appl. Anal. Discr. Math., 2009, vol. 3, no. 1, pp. 46-51.

[24] Neuman E., Sándor J. On certain means of two arguments and their extensions. Intern. J. Math. Sci., 2003, vol. 6, pp. 981-993

[25] Rado T. On convex functions. Trans. Amer. Math. Soc., 1935, vol. 37, pp. 266-285.

[26] Ruskeepää H. Mathematica ${ }^{\circledR}$ Navigator. 3rd ed. Academic Press, 2009.

[27] Sándor J. Two sharp inequalities for trigonometric and hyperbolic functions. Math. Inequal. Appl., 2012, vol. 15, no. 2, pp. 409-413.

[28] Sándor J. Some integral inequalities. Elem. Math., 1988, vol. 43, no. 6, pp. $177-180$.

[29] Sándor J. On two new means of two variables. Notes Number Th. Discr. Math., 2014, vol. 20, no. 1, pp. 1-9.

[30] Sándor J. On the identric and logarithmic means. Aequat. Math., 1990, vol. 40, pp. 261-270. DOI: 10.1007/BF02112299.

[31] Sándor J. A note on certain inequalities for means. Arch. Math. (Basel), 1991, vol. 56, pp. 471-473.

[32] Sándor J. On certain identities for means. Studia Univ. Babes-Bolyai, Math., 1993, vol. 38, pp. 7-14.

[33] Sándor J. On certain inequalities for means III. Arch. Math. (Basel), 2001, vol. 67, pp. 34-40.

[34] Sándor J. New refinements of two inequalities for means. J. Math. Ineq., 2013, vol. 2, no. 7, pp. 251-254.

[35] Sándor J. A note on log-convexity of the power means. Annales Math. Inf., 2015, vol. 45, pp. 107-110.

[36] Seiffert H. J. Comment to Problem 1365., Math. Mag., 1992, vol. 65, p. 356.

[37] Seiffert H. J. Ungleichungen für einen bestimmten Mittelwert. Nieuw Arch. Wisk. (Ser. 4), 1995, vol. 13, pp. 195-198.

[38] Seiffert H. J. Problem 88\%. Nieuw. Arch.Wisk., 1993, vol. 11, p. 176.

[39] Seiffert H. J. Ungleichungen für elementare Mittelwerte. Arch. Math. (Basel), 1995, vol. 64, pp. 129-131. 
[40] Vamanamurthy M. K., Vuorinen M. Inequalities for means. J. Math. Anal. Appl., 1994, vol. 183, pp. 155-166.

[41] Zhou S.-S., Qiam W.-M., Chu Y.-M., and Zhang X.-H. Sharp power-type Heronian mean bounds for Sándor and Yang means. J. Inequal. Appl., 2015, vol. 159. Doi:10.1186/s13660-015-0683-7.

Received November 21, $201 \%$.

In revised form, February 28, 2018.

Accepted February 28, 2018.

Published online March 31, 2018.

Babeş-Bolyai University

Str. Kogalniceanu nr. 1, 400084 Cluj-Napoca, Romania

E-mail: jsandor@math.ubbcluj.ro

Sukkur IBA University

Sindh, Pakistan

E-mail: barkat.bhayo@iba-suk.edu.pk 